\title{
Small Planar Monopole covers Multiband BRANs
}

\author{
M. J. Ammann * \& L. E. Doyle ** \\ Dublin Institute of Technology, Ireland * \\ Trinity College, Dublin, Ireland **

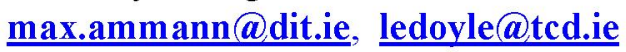

\begin{abstract}
The use of a single antenna which can cover a wide range of frequencies is a desirable feature for future wireless communications systems. The emerging self-organising and ad-hoc networks require more than an octave of bandwidth to facilitate multiband operation. A shorted planar monopole antenna is optimised for future wireless networking bands by suitable choice of planar dimension and feedgap separation. The antenna offers a return-loss greater than $10 \mathrm{~dB}$ from $1.60 \mathrm{GHz}$ to $5.90 \mathrm{GHz}$. The radiation pattern is shown to be quasiomnidirectional within the $10 \mathrm{~dB}$ return-loss bandwidth. Antenna properties are compared with the simple planar monopole.
\end{abstract}

\section{INTRODUCTION}

The proposed wideband antenna can cover future wireless data networking frequencies from $1.6 \mathrm{GHz}$ to $5.9 \mathrm{GHz}$. Included in this range of frequencies are DCS1800 (1.72 GHz to $1.88 \mathrm{GHz})$, BRANs (Broadband Radio Access Networks), $2.4 \mathrm{GHz}$ and $5.8 \mathrm{GHz}$ ISM bands and the US Unlicenced National Information Infrastructure band (U-NII) 5.15 - 5.35 $\mathrm{GHz}$ and 5.725 - $5.825 \mathrm{GHz}$. Also included are frequency bands employed by DECT (1.880-1.900 $\mathrm{GHz}$, PCS1900, UMTS, the European HiperLAN I, II and recent Bluetooth technology. A single wideband antenna covering these frequencies can be more attractive than multiband antennas operating in combinations of these bands.

\section{BACKGROUND}

The square planar monopole was first reported in 1976 , and radiation patterns were given over a range of frequencies [1]. Wideband planar disc antennas have been recently studied [2] and employed for the Japanese television band [3], but suffer from pattern distortion within the impedance bandwidth. The radiation pattern of the simple square monopole suffers no degradation within the impedance bandwidth. A planar monopole may be realised by replacing the wire element of a conventional monopole with a planar element as shown in Figure 1. By correct choice of geometry and feedgap separation, an impedance bandwidth ratio of $2.25: 1$ is easily achievable at $\mathrm{S}$ band.

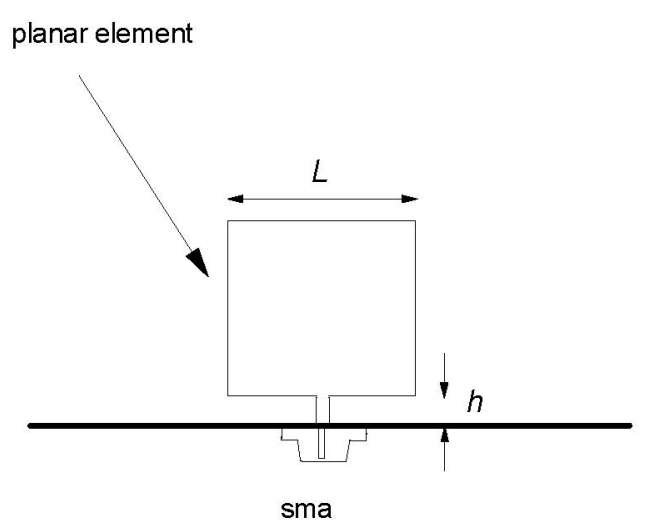

Figure 1. The simple square planar monopole is constructed using brass sheet $0.3 \mathrm{~mm}$ thick and fed through the groundplane using an SMA connector with a $1.2 \mathrm{~mm}$ diameter feedprobe. 
Measurements of the $10 \mathrm{~dB}$ return-loss bandwidth for various sizes of square monopole on a $150 \mathrm{~mm}$ groundplane, show a strong dependence on the feedgap separation [4]. A recently reported alteration to this antenna is to add a shorting post to one corner of the planar element [5]. This introduces an extra mode and reduces the lower edge frequency making the antenna more compact.

\section{ANTENNA DESIGN}

The shorted planar monopole has been optimised for this frequency range by choice of square dimension, $L$, and for maximum impedance bandwidth by choice of feedgap separation, $h$. The antenna was constructed from brass sheet, with the dimension, $L$, of the square planar element, of $25 \mathrm{~mm}$ and located centrally on a $150 \mathrm{~mm}$ square groundplane. Various feedgap separations, $h$, were investigated and a SMA connector with a $1.2 \mathrm{~mm}$ feedprobe diameter was employed. The shorting strip was $2.5 \mathrm{~mm}$ in width. (see Figure 2).

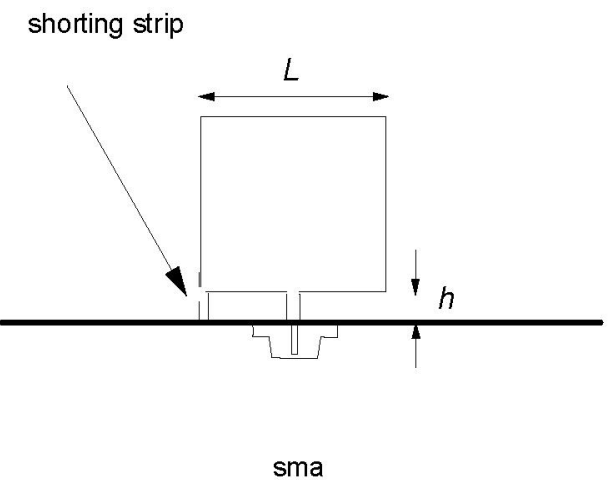

Figure 2. The modified square planar monopole with shorting plate and sma connector.

The antenna was accurately modelled using a method of moments analysis with a finite-gap feed and a wire-gridding technique was used for the planar element. This method has been shown to accurately predict the impedance bandwidth of planar monopole antennas for feedgaps greater than $1 \mathrm{~mm}$ [6], and to simulate the radiation patterns.

\section{IMPEDANCE BANDWIDTH FOR THE SIMPLE AND SHORTED PLANAR MONOPOLE}

Measurements of return loss have been made for a simple square planar monopole where the dimension $L$ of the square element is $25 \mathrm{~mm}$. The return-loss is shown to be greater than $10 \mathrm{~dB}$ over the frequency range $2.25 \mathrm{GHz}$ to $5.05 \mathrm{GHz}$ for the antenna with an optimised feedgap of $2.5 \mathrm{~mm}$ (see Figure 3). The addition of the shorting strip suggested in [5] was added to this structure and the bandwidth was found to increase further. However, the feedgap needs to be reduced to keep the return-loss greater than $10 \mathrm{~dB}$ in the midband, and this dimension is fairly critical. The optimum feedgap separation was found to be $1.7 \mathrm{~mm}$. The measured upper and lower edge frequencies for the shorted square element are $1.6 \mathrm{GHz}$ and $5.90 \mathrm{GHz}$ (see Figure 3). This represents an impedance bandwidth ratio of $3.7: 1$.

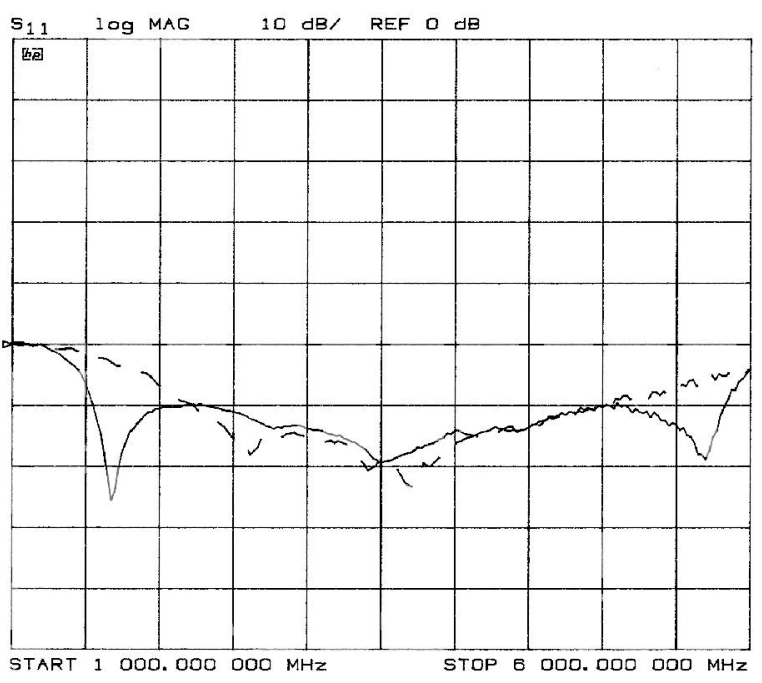

Figure 3. Measured return loss for the simple-square (dashed) and shorted-square (solid) monopoles show impedance bandwidth ratios of $2.25: 1$ and 3.7:1 respectively.

The swept return loss curves based on the wire grid equivalent of the planar element are shown to be in good agreement with experimental data. These are shown in Figure 4. 


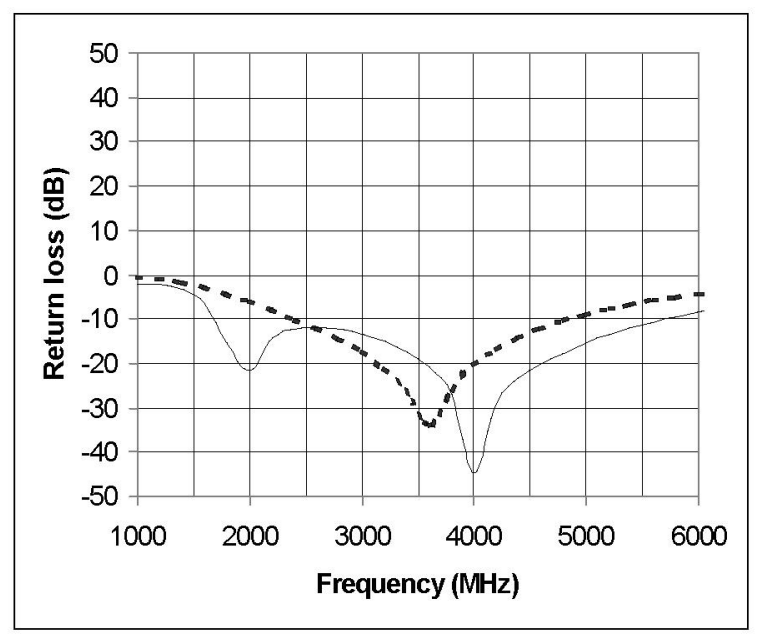

Figure 4. Calculated impedance bandwidth for the simple (dashed) and shorted (solid) square planar monopole, based on the MoM are in good agreement with experimental data.

\section{RADIATION PATTERN}

Both antennas exhibit quasi-omnidirectional patterns in the $\mathrm{H}$-plane with a maximum variation within the impedance bandwidth, of $\pm 1.8 \mathrm{~dB}$ and \pm $2.8 \mathrm{~dB}$ for the simple element and shorted-element respectively, when located on an infinite groundplane. These are shown in figures 6 and 7 . The difference between the patterns of the two elements is smaller when the groundplane is finite.

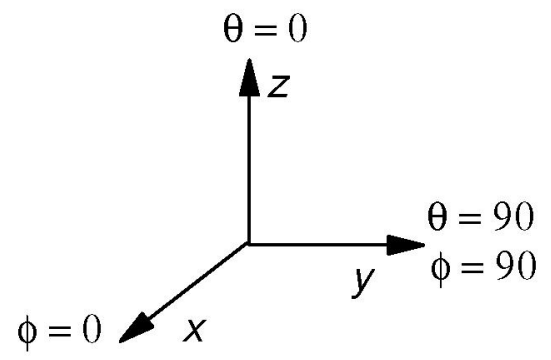

Figure 5. Coordinate system used. The planar element lies in the $y z(\phi=90)$ plane.

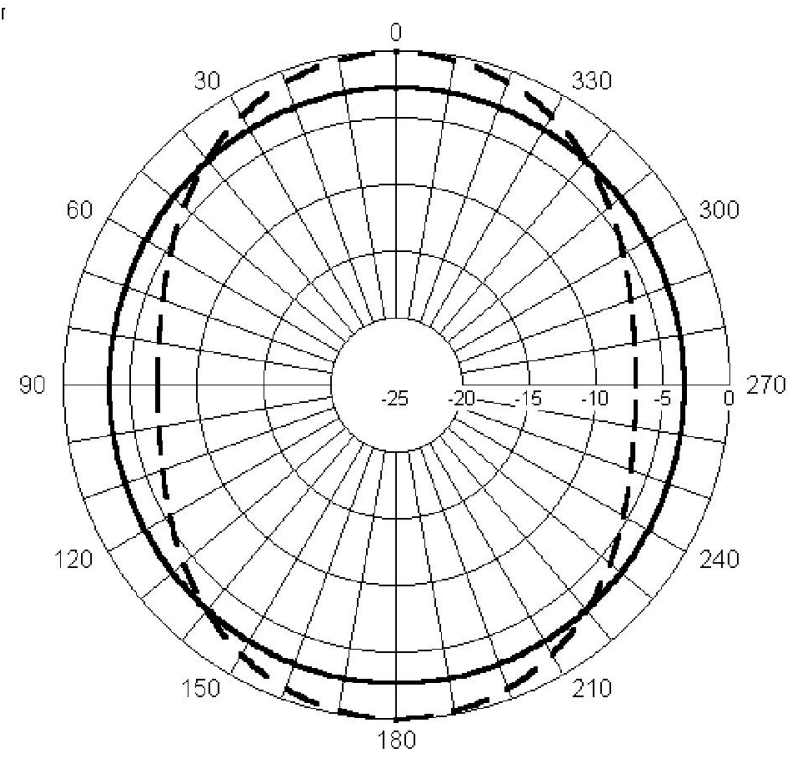

Figure 6. Normalised H-plane patterns [ $\phi, \theta=90$ ] for the shorted square planar monopole at frequencies of $2.4 \mathrm{GHz}$ (solid) and $5.8 \mathrm{GHz}$ (dashed), based on a method of moments computation

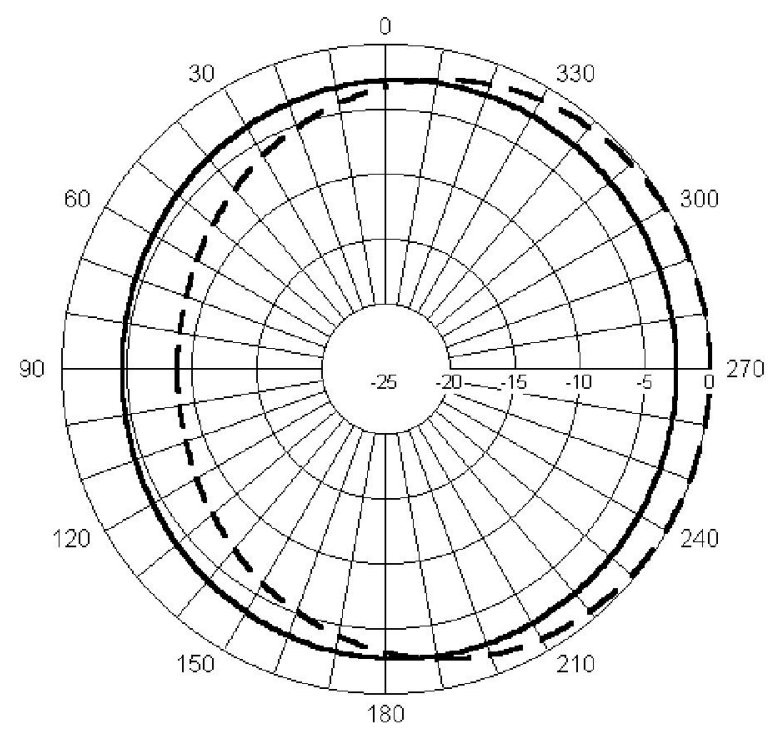

Figure 7. Normalised H-plane patterns [ $\phi, \theta=90]$ for the shorted square planar monopole at frequencies of $2.4 \mathrm{GHz}$ (solid) and $5.8 \mathrm{GHz}$ (dashed), based on a method of moments computation. 
The E-plane pattern displays monopolar patterns for both simple and shorted elements with a typical vertical null. The simple square planar monopole has a maximum gain of $4.5 \mathrm{dBi}$ at an elevation of 40 degrees with respect to the groundplane for $2.4 \mathrm{GHz}$ and at an elevation of 25 degrees at $5.8 \mathrm{GHz}$. The maximum gain of the shorted element is about $1.5 \mathrm{~dB}$ less than the simple element and the angles of elevation corresponding to maximum gain are the same for $2.4 \mathrm{GHz}$ and $5.8 \mathrm{GHz}$ given the same size groundplane.

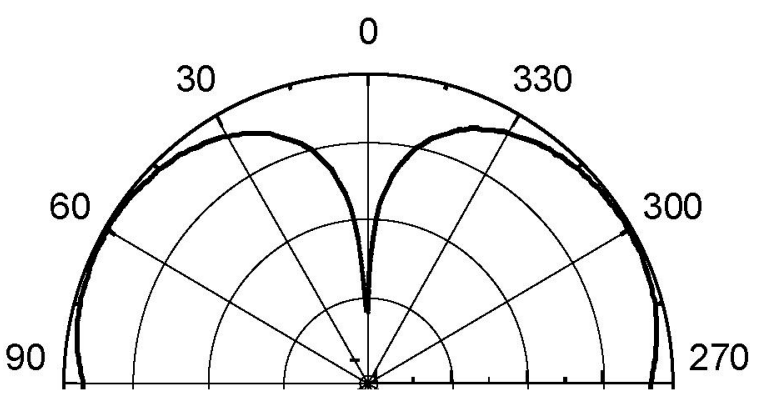

Figure 8. Normalised E-plane $[\theta, \phi=0]$ pattern for the simple planar monopole on a small groundplane (1 $\lambda$ square) at $2.4 \mathrm{GHz}$ based on a MoM/GTD prediction (10 $\mathrm{dB} / \mathrm{div})$.

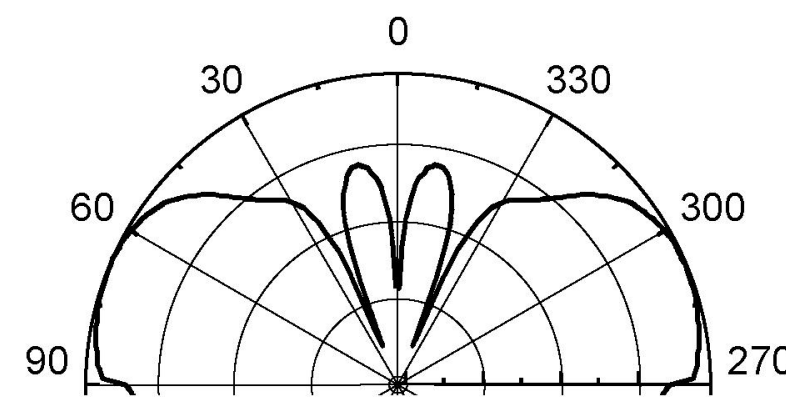

Figure 9. Normalised E-plane $[\theta, \phi=0]$ pattern for the simple planar monopole on a small groundplane $(2.5 \lambda$ square) at $5.8 \mathrm{GHz}$ based on a MoM/GTD prediction (10 $\mathrm{dB} / \mathrm{div})$.
The presence of extra lobes is observed at the upper end of the impedance bandwidth as shown in Figures 8 and 9. These patterns are in agreement with previous experimental work [4], and display typical monopolar groundplane dependency. The null depth and angle of elevation corresponding to maximum gain is groundplane dependent.

\section{CONCLUSION}

The shorted square monopole dimensions has been optimised to broaden the bandwidth to cover future demands in wireless data networks. A comparison has been made with the simple planar monopole. Both antennas are shown to have fairly frequency independent radiation patterns within the impedance bandwidth. The effect of feedgap distance is investigated and shown to be an important consideration.

\section{REFERENCES}

1. G. Dubost and S. Zisler, Antennas a large bande, Masson, Paris, New York, 1976, pp.128-129.

2. P. A. Agrawall, G. Kumar and K. Ray, 'Wideband Planar Monopole Antennas', IEEE Trans. Antennas and Propagation, 1998, AP-46, (2), 294-295.

3. S. Honda, M. Ito, H. Seki and Y. Jinbo, 'A Disk Monopole Antenna with 1:8 Impedance Bandwidth and Omnidirectional Radiation Pattern', Proc. ISAP, 1992 Sapporo, Japan, 1145-1148

4. M. J. Ammann, 'Square Planar Monopole Antennas' IEE Nat. Conf. Antennas \& Propagation, April 1999, York, UK, IEE Publication No. 461, pp. 37-40.

5. L. S. Lee, P. S. Hall and P. Gardner, 'Compact wideband planar monopole antenna' Electronics Letters. 1999, 35, pp. $2157-2158$

6. M. J. Ammann, 'Impedance Bandwidth of the Square Planar Monopole', Microwave and Optical Technology Letters, 2000, 24, (3), 185-187. 